\title{
Todes-Anzeige.
}

Am 23. Juli d. J. starb in Zürich der frühere Assistent der dortigen Sternwarte Dr. Johann Friedrich Höffler. Er war geboren zu Frankfurt a. M. am 28. April 1865 und als einziger Sohn einer dort alteingesessenen Kaufmannsfamilie zur Uebernahme der väterlichen Fabrik bestimmt. Doch gab der Vater den Vorstellungen seiner Lehrer an der dortigen Wöhlerschule nach und liess den Sohn $\times 88_{3}$ die Universität Giessen beziehen. Schon damals unterbrach häufige Kränklichkeit seine eifrig betriebenen Studien, doch konnte er I 886 nach Berlin übersiedeln und sich dort drei Jahre unter Foerster's Leitung eines ungehemmten Entwickelungsganges erfreuen. Dann aber zwang ihn 1889 die russische Influenza, in deren Gefolge tuberkulöse Erscheinungen auftraten, erst Falkenstein im Taunus, dann Davos aufzusuchen, unter gänzlicher Enthaltung von Arbeit. Erst 1894 setzte er an der Universität Zürich sein Studium fort und beendete es bald mit seiner lichtvollen Dissertation, Untersuchungen über die Existenz der objectiven Aberration. Ztirich $1895 \%$. Hier wird durch theoretische Entwickelungen der Nachweis erbracht, dass eine objective Aberration nicht existiren könne; d.h. der scheinbare Ort einer Lichtquelle ist unabhängig von einer etwaigen Bewegung derselben; und überdies weist er an der Berechnung von zwei Doppelsternbahnen nach, dass die erhebliche Deformation der Bahn, wie sie eine objective Aberration, wenn sie bestände, hervorrufen würde, nicht Platz greift. Höffler bekleidete dann die Stelle eines Assistenten der Sternwarte in Zürich, musste sie aber schon August 1896 wieder aufgeben, da seine schwankende Gesundheit nächtliche Beobachtungen nicht vertrug. Er blieb aber in Zürich mit theoretischen Arbeiten beschäftigt, deren eine Frucht er der Bamberger Astronomenversammlung vorlegte in dem Plane, aus den Verfinsterungen der Jupiterstrabanten die Richtung des Apex zu ermitteln, da der Weg für die von dort ausgehenden Lichtwellen bis zum Beobachter ein Minimum ist, wenn Jupiter in der Richtung des Apex steht, ein Maximum aber nach einem halben Jupitersumlauf. Wie er mir später mittheilte, hatte ein Versuch, das bisher vorliegende Beobachtungsmaterial in diesem Sinne auszuwerthen, wegen dessen Ungenauigkeit kein positives Ergebniss gehabt. Seine letzte Arbeit ist dann den Lesern der Astr. Nachr. bekannt aus Nr. $345^{6}$ in dem Versuch einer Bestimmung der Parallaxe des Systems Ursa major *. Er gelangt hier ganz selbstständig dazu, eine früher schon von Klinkerfues geäusserte, aber fast unbeachtet gebliebene Idee mit dem bessern Material, das jetzt verfügbar ist, zu einem höchst interessanten Ergebniss zu führen. Aber wiederum wurde er ans Krankenbett gefesselt, wie der Arzt bald erkannte, durch ein unheilbares melanosarkomatisches Leiden, wie Höffler selbst bis an sein Ende glaubte, durch Gelenkrheumatismus. So sah ich ihn noch am 9. Juni d.J. in dem herrlich gelegenen Spital zum Rothen Kreuz in Zürich-Fluntern, die geistvollen Züge schmerzverklärt, von neuen Arbeiten sprechend, die er nach der bald erhofften Genesung zu vollenden dachte und unter denen Untersuctungen tiber die Eigenbewegung des Sonnensystems obenan standen. Nach sechs Wochen indess hatte die unerbittliche Krankheit all diesen Entwürfen ein Ziel gesetzt und das Leben eines Dulders geendet, der den wenigen gesunden Tagen, die ihm vergönnt waren, so schöne Arbeiten abgerungen. Sie zeugen von einem Genius, der seinen Hingang im Alter von kaum 33 Jahren für unsere Wissenschaft zu einem tief beklagenswerthen macht.

Kiel 1898 Oct. 14 .

F. Ristenpart.

\section{Numerirung der kleinen Planeten 1897 DL bis 1898 DR.}

Von den neuen kleinen Planeten sind folgende mit Nummern versehen worden:

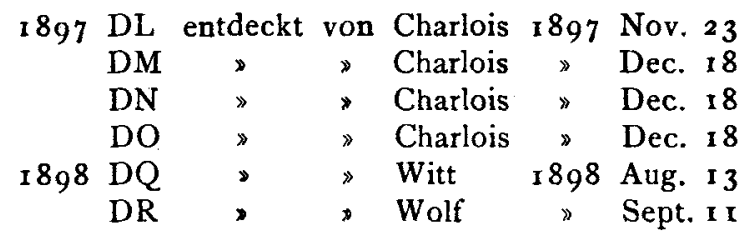

$(429)$

(434) Hungaria

$1898 \mathrm{DP}$, entdeckt von Charlois 1898 Juli 16 , ist vorläufig von der Numerirung ausgeschlossen worden, da das veröffentlichte Beobachtungsmaterial zu einer Bahnbestimmung nicht ausreicht.

Berlin, Kgl. Astronomisches Recheninstitut, 1898 Oct. 26.

Ұ. Bauschinger.

Inhalt zu Nr. 3526. F. Schreiber, S. J. Ueber eine eigenthiumliche Gangperiode einer Pendeluhr. 353. - A. Berberich. Ueber die Störungen der Bahn des Leonidenschwarmes seit 1890. 359. - A. Wolfer. Ueber den Inhalt der Nr. 89 der Astronomischen Mittheilungen. 36r. - W. Schur. Vergleichsterne zu den Beobachtungen der grossen Planeten. 361. - E. C. Pickering. Harvard College Observatory Circular No. 34. 363. - E. Millosevich. Elementi ellittici del pianeta (433) (1898 DQ). 363. - Beobachtungen des Cometen $1898 \ldots$...(Brooks Oct. 20). 365. - Elemente und Ephemeride des Cometen I898...(Brooks Oct. 20). 365. V. Cerulli. Correctionen von Planeten-Ephemeriden. 365. - F. Ristenpart. Anzeige des Todes von Johann Friedrich Höffler. 367. - F. Bauschinger. Numerirung der kleinen Planeten 1897 DL bis 1898 DR. 367 . 